\title{
Correction to: Wellposedness and Convergence of Solutions to a Class of Forced Non-diffusive Equations with Applications
}

Susan Friedlander and Anthony Suen

\section{Correction to: J. Math. Fluid Mech. (2019) 21:50}

\section{https://doi.org/10.1007/s00021-019-0454-1}

In this erratum we revise the hypothesis and statement of [1, Theorem 2.3] to prove local in time existence of analytic, rather than Gevrey class solutions. As a consequence, we also revise the results in $[1$, Theorem 2.5] for the convergence of analytic solutions as $\nu$ goes to zero.

To begin with, we first recall that the abstract active scalar equations which are given by

$$
\left\{\begin{array}{l}
\partial_{t} \theta^{\nu}+u^{\nu} \cdot \nabla \theta^{\nu}=S \\
u_{j}^{\nu}=\partial_{x_{i}} T_{i j}^{\nu}\left[\theta^{\nu}\right], \theta^{\nu}(x, 0)=\theta_{0}(x)
\end{array}\right.
$$

where $\mathbb{T}^{d} \times(0, \infty)=[0,2 \pi]^{d} \times(0, \infty)$ with $d \geq 2$. We assume that

$$
\int_{\mathbb{T}^{d}} \theta^{\nu}(x, t) d x=\int_{\mathbb{T}^{d}} S(x)=0 \text { for all } t \geq 0
$$

The symbols $\left\{T_{i j}^{\nu}\right\}_{\nu \geq 0}$ refer to a sequence of operators which satisfy:

A1 $\partial_{i} \partial_{j} T_{i j}^{\nu} f=0$ for any smooth functions $f$ for all $\nu \geq 0$.

A2 $T_{i j}^{\nu}: L^{\infty}\left(\mathbb{T}^{d}\right) \rightarrow B M O\left(\mathbb{T}^{d}\right)$ are bounded for all $\nu \geq 0$.

A3 For each $\nu>0$, there exists a constant $C_{\nu}>0$ such that for all $1 \leq i, j \leq d$,

$$
\left|\widehat{T}_{i j}^{\nu}(k)\right| \leq C_{\nu}|k|^{-3}, \forall k \in \mathbb{Z}^{d}
$$

A4 For each $1 \leq i, j \leq d$,

$$
\lim _{\nu \rightarrow 0} \sum_{k \in \mathbb{Z}^{d}}\left|\widehat{T_{i j}^{\nu}}(k)-\widehat{T_{i j}^{0}}(k)\right|^{2}|\widehat{g}(k)|^{2}=0
$$

for all $g \in L^{2}$.

Moreover, we further assume that $\left\{T_{i j}^{\nu}\right\}_{\nu \geq 0}$ satisfy either one of the following assumptions:

A5 1 There exists a constant $C_{0}>0$ independent of $\nu$, such that for all $1 \leq i, j \leq d$,

$$
\begin{gathered}
\sup _{\nu \in(0,1]} \sup _{\left\{k \in \mathbb{Z}^{d}\right\}}\left|\widehat{T}_{i j}^{\nu}(k)\right| \leq C_{0} ; \\
\sup _{\left\{k \in \mathbb{Z}^{d}\right\}}\left|\widehat{T}_{i j}^{0}(k)\right| \leq C_{0} .
\end{gathered}
$$

The original article can be found online at https://doi.org/10.1007/s00021-019-0454-1. 
$\mathrm{A} 55_{2}$ There exists a constant $C_{0}>0$ independent of $\nu$, such that for all $1 \leq i, j \leq d$,

$$
\begin{gathered}
\sup _{\nu \in(0,1]} \sup _{\left\{k \in \mathbb{Z}^{d}\right\}}\left|k_{i} \widehat{T}_{i j}^{\nu}(k)\right| \leq C_{0} ; \\
\sup _{\left\{k \in \mathbb{Z}^{d}\right\}}\left|k_{i} \widehat{T}_{i j}^{0}(k)\right| \leq C_{0} .
\end{gathered}
$$

We now give the revised statement for Theorem 2.3 given in [1]:

Theorem 2.3. (Analytic local wellposedness in the case $\nu=0$ ). Fix $r>\frac{d}{2}+\frac{3}{2}$ and $K_{0}>0$. Let $\theta^{0}(\cdot, 0)=\theta_{0}$ and $S$ be analytic functions with radius of convergence $\tau_{0}>0$ and satisfy

$$
\left\|\Lambda^{r} e^{\tau_{0} \Lambda} \theta^{0}(\cdot, 0)\right\|_{L^{2}} \leq K_{0}, \quad\left\|\Lambda^{r} e^{\tau_{0} \Lambda} S\right\|_{L^{2}} \leq K_{0} .
$$

For $\nu=0$, under the assumptions A1-A2 and $A 5_{1}$, there exists $\bar{T}, \bar{\tau}>0$ and a unique analytic solution $\theta^{0}$ to (1.1) defined on $\mathbb{T}^{d} \times[0, \bar{T}]$ with radius of convergence at least $\bar{\tau}$. In particular, there exists a constant $C=C\left(K_{0}\right)>0$ such that for all $t \in[0, \bar{T}]$,

$$
\left\|\Lambda^{r} e^{\bar{\tau} \Lambda} \theta^{0}(\cdot, t)\right\|_{L^{2}} \leq C .
$$

Moreover, if the assumption A3 holds as well, then we have

$$
\left\|\Lambda^{r} e^{\bar{\tau} \Lambda} \theta^{\nu}(\cdot, t)\right\|_{L^{2}} \leq C, \forall \nu>0,
$$

where $\theta^{\nu}$ are analytic solutions to (1.1) for $\nu>0$ as described in [1, Theorem 2.2].

Proof of Theorem 2.3. The results follow by the similar argument given by Friedlander and Vicol [2] for the unforced system with $S \equiv 0$ in (1.1).

We also revise the statement of [1, Theorem 2.5] for the convergence of analytic solutions to (1.1).

Theorem 2.5. (Convergence of solutions as $\nu \rightarrow 0$ ). Depending on the assumptions $A 5_{1}$ and $A 5_{2}$, we have the following cases:

- Assume that the hypotheses and notations of Theorem 2.3 are in force. Under the assumptions A3$A 4$, if $\theta^{\nu}$ and $\theta^{0}$ are analytic solutions to (1.1) for $\nu>0$ and $\nu=0$ respectively with initial datum $\theta_{0}$ on $\mathbb{T}^{d} \times[0, \bar{T}]$ with radius of convergence at least $\bar{\tau}$ as described in Theorem 2.3, then there exists $T<\bar{T}$ and $\tau=\tau(t)<\bar{\tau}$ such that, for $t \in[0, T]$, we have

$$
\lim _{\nu \rightarrow 0}\left\|\left(\Lambda^{r} e^{\tau \Lambda} \theta^{\nu}-\Lambda^{r} e^{\tau \Lambda} \theta^{0}\right)(\cdot, t)\right\|_{L^{2}}=0 .
$$

- Assume that the hypotheses and notations of [1, Theorem 2.4] are in force. Under the assumptions $A 3-A 4$, for $d \geq 2$ and $s>\frac{d}{2}+1$ and $t \in[0, T]$, we have

$$
\lim _{\nu \rightarrow 0}\left\|\left(\theta^{\nu}-\theta^{0}\right)(\cdot, t)\right\|_{H^{s-1}}=0 .
$$

Remark 2.6. The proof for the convergence result (2.7) follows by the same argument given in [1, pp. 16$18]$ by taking $s=1$.

Remark 2.7. The applications to the magnetostrophic equations given in [1, Sect. 6] now hold under the revised statements of Theorems 2.3 and 2.5.

Acknowledgements. S. Friedlander is supported by NSF DMS-1613135 and A. Suen is supported by Hong Kong General Research Fund (GRF) grant project number 18300720.

\section{Compliance with ethical standards}

Conflict of interest The authors declare that they have no conflict of interests.

Publisher's Note Springer Nature remains neutral with regard to jurisdictional claims in published maps and institutional affiliations. 


\section{References}

[1] Friedlander, S., Suen, A.: Well-posedness and convergence of solutions to a class of forced non-diffusion equations. J. Math. Fluid Mech. 21(4), 21-50 (2019)

[2] Friedlander, S., Vicol, V.: On the ill/wellposedness and nonlinear instability of the magnetogeostrophic equations. Nonlinearity 24(11), 3019-3042 (2011)

Susan Friedlander

Department of Mathematics

University of Southern California

Los Angeles

USA

e-mail: susanfri@usc.edu
Anthony Suen

Department of Mathematics and Information Technology

The Education University of Hong Kong

Tai Po

Hong Kong

e-mail: acksuen@eduhk.hk

(accepted: June 23, 2021; published online: July 20, 2021) 